УДК $551.510 .532 ; 551.501 .816$

\title{
ЛИДАР ДЛЯ ИЗМЕРЕНИЯ ВЕРТИКАЛЬНОГО РАСПРЕДЕЛЕНИЯ ОЗОНА В АТМОСФЕРЕ
}

\author{
Долгий Сергей Иванович', \\ dolgii@iao.ru
}

\author{
Невзоров Алексей Алексеевич', \\ naa@iao.ru
}

\section{Невзоров Алексей Викторович', nevzorov@iao.ru}

\section{Романовский Олег Анатольевич1,2, roa@iao.ru}

\author{
Харченко Ольга Викторовна', \\ olya@iao.ru \\ 1 Институт оптики атмосферы им. В.Е. Зуева Сибирского отделения Российской академии наук, \\ Россия, 634021, г. Томск, пл. Академика Зуева, 1. \\ 2 Национальный исследовательский Томский государственный университет, \\ Россия, 634050, г. Томск, пр. Ленина, 36.
}

Одним из основных климатообразующих факторов с точки зрения радиационного баланса атмосферы являются парниковые газы, прежде всего озон и газовые составляющие озоновых циклов. В настоящее время оперативную информацию об их вертикальном распределении в атмосфере можно получать только с использованием средств дистанционного зондирования, в том числе лазерного зондирования (лидаров), что определяет актуальность и научную значимость данной научной и технической проблемы.

Цель: разработка лидара для исследований вертикального распределения озона в верхней тропосфере-стратосфере в высотном диапазоне (5-45) км на длинах волн 299/341 и 308/353 нм в сопоставлении со спутниковыми измерениями.

Методы: метод дифференциального поглощения и рассеяния.

Результаты. Представлен лидар для измерения вертикального распределения озона над городом Томск (56,5 с.ш.; 85,0 в.д.). Лидарное зондирование озона производилось на парах длин волн 299/341 и 308/353 нм. Используемые длины волн позволяют проводить мониторинг вертикальной стратификации озона в пределах верхней тропосферы-стратосферы. Результаты лидарного зондирования озона по данным января 2018 г. приведены в сравнении с данными спутников IASI/MetOp и AURA, а также с среднеширотной моделью Крюгера. Отличие лидарных профилей озона от модельных является естественными для динамики озонового слоя в различные дни наблюдений. Большие различия вертикального распределения озона в значениях по данным лидара и спутника МеtОр на высотах стратосферы объясняются необходимостью коррекции априорной модели в алгоритме восстановления спутниковых данных.

Вывод. Создан лидар для измерения озоновых профилей в верхней тропосфере-стратосфере. Он позволяет восстанавливать вертикальное распределение озона в высотном диапазоне (5-45) км на длинах волн 299/341 и $308 / 353$ нм.

\section{Ключевые слова:}

Лидар, озон, атмосфера, дифференциальное поглощение, спутник MetOp, спутник AURA.

\section{Введение}

В настоящее время представляется актуальным рассмотрение вопросов техники и технологии прогнозирования состояния озонового слоя атмосферы, представляющего собой ценный георесурс, незаменимый для обеспечения комфортного состояния окружающей среды и решения проблем климатологии планеты.

Одним из основных климатообразующих факторов с точки зрения радиационного баланса атмосферы являются парниковые газы, прежде всего озон и газовые составляющие озоновых циклов. Изучение газового состава атмосферы без применения дистанционных технологий представляется весьма затруднительным. В настоящее время оперативную информацию о вертикальном распределении газовых компонент в атмосфере можно получать только с использованием средств дистанционного зондирова- ния, что определяет актуальность и научную значимость данной научной и технической проблемы.

Среди дистанционных технологий важное место занимают лазерные методы зондирования, разрабатываемые с момента изобретения лазера в 1961 г. Лидары (лазерные локаторы) используют различные эффекты взаимодействия световых импульсов с атмосферной средой и показали высокую эффективность при определении физических характеристик (температура, давление, ветер), а также содержания газовых примесей от поверхности земли до высот 80-100 км [1-10]. При этом возможности лидаров обеспечивают изучение атмосферных процессов в масштабах от нескольких кубических метров и нескольких секунд до глобальных с многолетними рядами данных.

В настоящее время лазерное зондирование озоносферы приобрело регулярный характер [10-18]. 
Таблица 1. Лидарные комплексы для измерения вертикального распределения озона

Table 1. Lidar complexes for measuring ozone vertical distribution (OVD)

\begin{tabular}{|c|c|c|c|c|c|c|c|c|}
\hline $\begin{array}{c}\text { Станция } \\
\text { Station }\end{array}$ & $\begin{array}{l}\text { Лазер } \\
\text { Laser }\end{array}$ & $\begin{array}{l}\text { Длина волн, нм } \\
\text { Wavelength, nm }\end{array}$ & $\begin{array}{l}\text { BKP } \\
\text { SRS }\end{array}$ & $\begin{array}{l}\text { Пары длин волн, нм } \\
\text { Wavelength pairs, nm }\end{array}$ & $\begin{array}{c}\text { Диапазон высот, км } \\
\text { Altitude range, km }\end{array}$ & \begin{tabular}{|c|} 
Ошибки \\
Errors, \%
\end{tabular} & $\begin{array}{c}\text { Зеркало, м } \\
\text { Mirror, m }\end{array}$ & $\begin{array}{l}\text { Источник } \\
\text { Links }\end{array}$ \\
\hline $\begin{array}{l}\text { Цукуба } \\
\text { Tsukuba }\end{array}$ & $\begin{array}{c}\text { Nd: YAG } \\
\text { XeCl } \\
\text { Nd: YAG } \\
\text { XeF }\end{array}$ & $\begin{array}{l}266 \\
308 \\
355 \\
351\end{array}$ & $\begin{array}{c}\mathrm{CO}_{2} \\
\mathrm{D}_{2}\end{array}$ & $\begin{array}{l}276 / 287 \\
287 / 299 \\
308 / 355 \\
308 / 351 \\
308 / 339\end{array}$ & $\begin{array}{c}0,4-3 \\
3-10 \\
15-45 \\
10-45 \\
10-45\end{array}$ & $\begin{array}{c}3-9 \\
5-30\end{array}$ & $\begin{array}{c}0,25 \\
0,6 \\
1 \\
1 \\
2\end{array}$ & {$[10,11]$} \\
\hline $\begin{array}{c}\text { Верхний } \\
\text { Прованс } \\
\text { ОНР }\end{array}$ & $\begin{array}{c}\text { Nd: YAG } \\
\text { XeCl } \\
\text { Nd: YAG }\end{array}$ & $\begin{array}{l}266 \\
308 \\
355\end{array}$ & $\begin{array}{c}\mathrm{D}_{2} \\
-\end{array}$ & $\begin{array}{l}289 / 316 \\
308 / 355\end{array}$ & $\begin{array}{c}3-14 \\
15-45\end{array}$ & $\begin{array}{c}10 \\
5-20\end{array}$ & $\begin{array}{c}0,4 \\
4 \text { шт. } \\
0,53\end{array}$ & {$[12,13]$} \\
\hline $\begin{array}{c}\text { Столовая гора } \\
\text { TMF }\end{array}$ & $\begin{array}{c}\text { Nd: YAG } \\
\text { XeCl } \\
\text { Nd: YAG }\end{array}$ & $\begin{array}{l}266 \\
308 \\
355\end{array}$ & $\begin{array}{c}\mathrm{D}_{2} \\
\mathrm{H}_{2} \mathrm{H}_{2}\end{array}$ & $\begin{array}{l}289 / 299 \\
308 / 353\end{array}$ & $\begin{array}{c}3-18 \\
15-50\end{array}$ & $\begin{array}{l}7-14 \\
5-30\end{array}$ & $\begin{array}{c}0,91 \\
0,9\end{array}$ & {$[14,15]$} \\
\hline $\begin{array}{c}\text { ЦКП им. Годдарда } \\
\text { GSFC }\end{array}$ & $\begin{array}{c}\text { Nd: YAG } \\
\text { XeCl } \\
\text { Nd: YAG }\end{array}$ & $\begin{array}{l}266 \\
308 \\
355\end{array}$ & $\begin{array}{c}\mathrm{D}_{2} \\
\mathrm{H}_{2} \\
-\end{array}$ & $\begin{array}{l}289 / 299 \\
308 / 355\end{array}$ & $\begin{array}{c}1,5-12 \\
10-50\end{array}$ & $\begin{array}{c}16-19 \\
5-30\end{array}$ & $\begin{array}{l}0,45 \\
0,76\end{array}$ & {$[16,17]$} \\
\hline $\begin{array}{c}\text { Владивосток } \\
\text { Vladivostok }\end{array}$ & $\mathrm{XeCl}$ & 308 & $\mathrm{H}_{2}$ & $308 / 353 / 331$ & $5-40$ & $2-30$ & 0,6 & [18] \\
\hline
\end{tabular}

В табл. 1 представлены основные характеристики лидарных комплексов для следующих станций: Tsukuba (36.05 с.ш., $140.13^{\circ}$ в.д.), Япония [10, 11]; Observatoire de Haute Provence (OHP) (43.94 ${ }^{\circ}$ с.ш., $5.71^{\circ}$ в.д.), Франция [12, 13]; Table Mountain Facility (TMF) (34.4 с.ш., $117.7^{\circ}$ з.д.), США [14, 15]; Goddard Space Flight Center (GSFC) или Центр космических полетов имени Годдарда (ЦКП им. Годдарда) (37.1 с.ш, $76.39^{\circ}$ з.д.), США [16, 17]; Владивосток (43.3ㄷ.ш., $132^{\circ}$ в.д.), Россия [18].

Натурные эксперименты в стратосфере на Сибирской лидарной станции (СЛС) [5] (г. Томск: $56,5^{\circ}$ с.ш.; 85, $0^{\circ}$ в.д.) показали, что при применении пары длин волн 308/353 нм максимальная высота потолка зондирования стратосферы достигает $\sim 40-45$ км (озоновый максимум в Томске расположен в высотном интервале 19-21 км), учитывая, что нижняя граница зондирования составляет 15 км [19].

На СЛС также проводится лидарное зондирование озона в верхней тропосфере-нижней стратосфере на паре длин волн 299/341 нм. Эксперименты показали, что при применении этих длин волн охват высот составляет $\sim 6-15$ км [20], а в удачных случаях охват высот достигает диапазона $\sim 5-20 \mathrm{kм}$.

Целью данной работы является создание лидара для измерения вертикального распределения озона (ВРО) в верхней тропосфере-стратосфере в высотном диапазоне (5-45) км на длинах волн 299/341 и $308 / 353$ нм в сопоставлении со спутниковыми измерениями. Лидар для измерений озона в диапазоне высот 5-45 км позволит выявлять изменчивость ВРО в верхней тропосфере-стратосфере, где озон играет роль пассивного трассера циркуляционных процессов, и на высотах выше велопаузы ( 26 км), где поведение озоносферы определяется фотохимическими процессами.

\section{Аппаратура}

Для расширения возможностей изучения особенностей ВРО в верхней тропосфере-стратосфере, особенно в области локализации озонового слоя, и отслеживания его сезонной изменчивости модернизирован и введен в режим регулярных измерений лидар для измерений ВРО в высотном диапазоне (5-45) км на длинах волн 299/341 и 308/353 нм. Попеременное лидарное зондирование озоносферы на парах длин волн 299/341 и 308/353 нм позволяет охватить в наиболее удачных измерениях диапазон высот от $\sim 5$ до $\sim 45$ км.

На рис. 1 представлена блок-схема созданного лидара для зондирования озона в верхней тропосфер-стратосфере.

Основные технические характеристики лазерных источников и приемных оптических элементов лидарной системы:

$$
\text { Передатчик }
$$

Длина волны зондирования, $\lambda$ нм Энергия импульса, мДж (соотв. $\lambda$ )

Частота следования, Гц (соотв. $\lambda$ )

Расходимость, мрад

$\begin{array}{cc}\text { Nd: YAG } & \mathrm{XeCl} \\ 299 / 341 & 308 / 353 \\ 25 / 20 & 100 / 50 \\ 15 & 100 \\ 0,1-0,3 & 0,1-0,3 \\ \text { Tелескоп } & \\ \text { системы } & \\ \text { Ньютона } & \\ & \end{array}$

Приемник

0,5

\section{Диаметр зеркала, м}

1,5

В качестве источников излучения лидарной системы для зондирования озона в верхней тропосфере - нижней стратосферы используется $\mathrm{Nd}$ : YAG-лазер - модель LS-2134UT Минской фирмы «LOTIS TII», работающий на длине волны 266 нм. Для измерений стратосферного озона используется эксимерный $\mathrm{XeCl}$ лазер LPX-120i фирмы «Lambda Physik», работающий на длине волны 308 нм. Излучения лазеров, проходя через ячейки ВКР преобразования, попадают на автоматизированные поворотные зеркала (ПЗ), которые упра- 


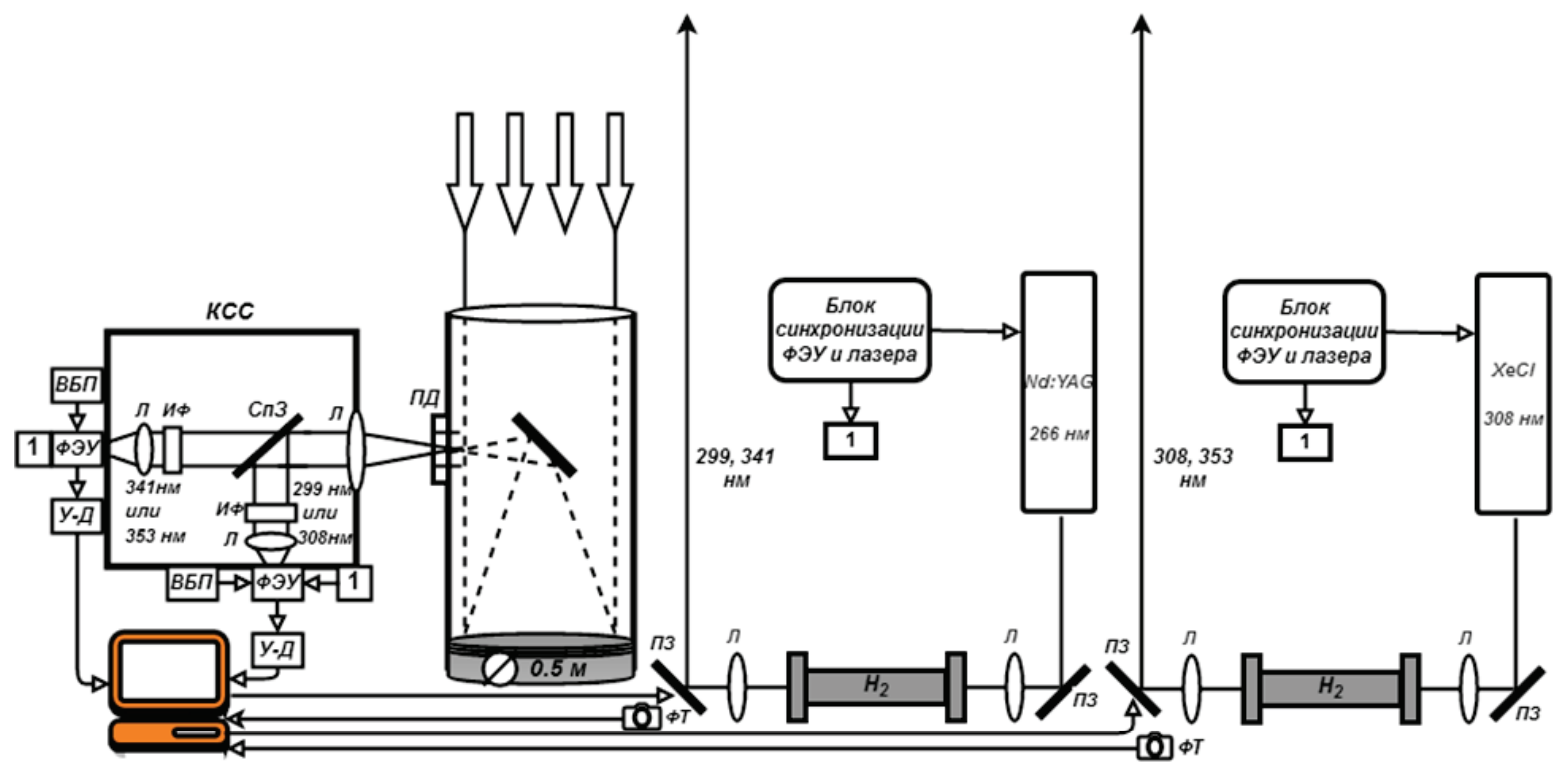

Pис. 1. Блок-схема озонового лидара: ПД - полевая диафрагла; КСС - кювета спектральной селекиии с ФЭУ; СпЗ - спектроделительное зеркало; УД - усилители-дискриминаторы; ВБП - высоковольтные блоки питания; ПЗ - поворотные зеркала; л -линзы; КСС - кювета спектральной селекции; ИФ - интерференционные фильтры; ФТ - фототранзистор

Fig. 1. Block diagram of the ozone lidar: ПД (FD) - field diaphragm; KCC (CSS) - cell of spectral selection with a photomultiplier; Cn3 (SM) spectrodeliver mirror; УД (AD) - amplifiers-discriminators; ВБП (HVPS) - high-voltage power supplies; ПЗ (RM) - rotary mirrors; $\pi(L)$ - lenses; $И Ф(I F)$ - interferential filters; $\Phi T(F T)$ - phototransistor

вляются от компьютера и выводятся в атмосферу. Прием оптических сигналов ведется на телескоп с диаметром зеркала 0,5 м. После телескопа оптическое излучение попадает в кювету спектральной селекции (КСС), где происходит выделение по длинам волн зондирования.

Для тропосферных измерений используется оптика, работающая на длинах волн 299/341 нм, для стратосферных - 308/353 нм, соответственно. Далее оптическое излучение фокусируется линзами (Л) на фотоэлектронные умножители (ФЭУ) R7207-01 фирмы HAMAMATSU, работающие в режиме счета импульсов фототока. Чтобы устранить искажения, обусловленные обратно-рассеянными сигналами высокой интенсивности от ближней зоны зондирования или облаков, блоки ФЭУ оснащены электронным управлением коэффициента усиления. Электрические сигналы с ФЭУ поступают на усилители-дискриминаторы (У-Д), где происходит их усиление и формирование импульсов до уровней +5 вольт. Сформированные электрические сигналы с У-Д поступают на соответствующие каналы регистратора импульсов (счетчик фотонов), который осуществляет накопление и запись лидарных сигналов в цифровом виде на жесткий диск компьютера. Время измерения стратосферного озона составляет $20-40$ минут. Перенастройка приемника для регистрирования другой пары длин волн примерно 1-5 минут. Время измерения озона на паре 299/341 нм составляет $\sim 40$ минут. Обработка результатов измерений проводится с помощью методики восстановления лидарных профилей ВРО с учетом температурной и аэрозольной коррекции.
Результаты лидарных измерений сопоставлялись с результатами измерений, полученными с помощью микроволнового радиометра MLS, установленного на борту метеорологического спутника AURA, а также интерферометра IASI, установленного на борту метеорологического спутника MetOpt.

Микроволновой радиометр MLS, производящий мониторинг атмосферных составляющих $\mathrm{BrO}$, $\mathrm{CH}_{3} \mathrm{Cl}, \mathrm{CO}, \mathrm{ClO}, \mathrm{HCl}, \mathrm{HNO}_{3}, \mathrm{HO}_{2}, \mathrm{~N}_{2} \mathrm{O}, \mathrm{O}_{3}$ и др., а также профилей температуры и влажности в стратосфеpe в рамках американской программы NASA Earth Observing System, расположен на борту американского научно-исследовательский спутника AURA. Используются стандартные озоновые продукты данных (240 ГГц излучение), восстановленные с использованием 4,2 версии алгоритма обработки данных, которые свободно публикуются [21, 22].

Интерферометр IASI расположен на борту метеорологического спутника Европейского космического агентства (MetOp), ведущего измерения атмосферных составляющих $\mathrm{CO}_{2}, \mathrm{CH}_{4}, \mathrm{~N}_{2} \mathrm{O}, \mathrm{CO}, \mathrm{O}_{3}$, $\mathrm{HNO}_{3}$, а также профилей температуры и влажности в тропосфере и нижней стратосфере в рамках европейской программы European Polar System в режиме, близком к режиму реального времени. IASI обеспечивает получение спектров высокого радиометрического качества с разрешением $0,5 \mathrm{~cm}^{-1}$ в диапазоне от 625 до $2760 \mathrm{~cm}^{-1}$ [23]. Восстановление профилей озона по данным спутникового зондирования выполняется в диапазоне $1025-1075 \mathrm{~cm}^{-1}$.

Спутниковые данные IASI получены с помощью станции приёма спутниковой информации 2,4 XLB фирмы Orbital Systems (США), введённой 
в эксплуатацию в ИОА СО РАН в 2011 г. [20]. Спутниковые данные MLS находятся в свободном доступе на сайте NASA [22]. Получаемая информация позволяет проводить сравнение спутниковых данных с результатами лидарного зондирования на СЛС.

\section{Методика измерений и анализ ошибок}

Наиболее чувствительным из методов, используемых для определения пространственного распределения концентрации какого-либо атмосферного газа, в том числе и озона, является метод дифференциального поглощения (MДП или DIAL). В основу методики восстановления лидарных профилей ВРО с учетом температурной и аэрозольной коррекции легло уравнение [24]:

$$
\begin{aligned}
& n(H)=\underbrace{\frac{1}{2\left[k_{o n}(H)-k_{\text {off }}(H)\right]}}_{A} \times
\end{aligned}
$$

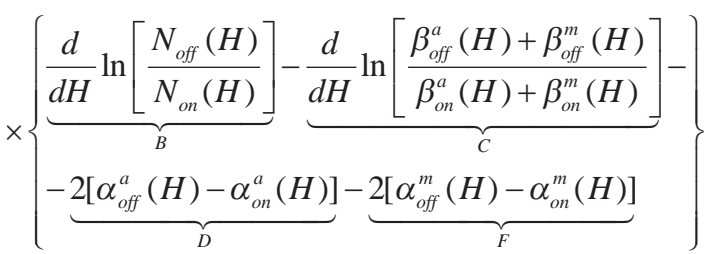

где $N(H)$ - зарегистрированный эхо-сигнал в линии поглощения - on и вне линии поглощения off; $\alpha^{a}$ - коэффициент ослабления аэрозольного рассеяния; $\beta^{a}$ - коэффициент обратного аэрозольного рассеяния; $k$ - коэффициент поглощения; $n$ $(H)$ - концентрация озона.

Реальные вариации температуры в атмосфере могут вызвать существенные изменения в априорном расчете коэффициента поглощения озона, что приводит к систематическим ошибкам восстановления профилей ВРО. Поэтому в алгоритме восстановления ВРО целесообразно проводить коррекцию на температурную зависимость $k_{\text {on }}(H, T)$, $k_{\text {off }}(H, T)$. В предложенной нами методике использована зависимость сечения поглощения озона от температуры, полученная по последним экспериментальным и расчетным данным из работ $[25,26]$. Эта зависимость представлена в табл. 2.

При больших значениях аэрозольного наполнения атмосферы обратное аэрозольное рассеяние в несколько раз превышает молекулярное, что при неучтенных рассеивающих и ослабляющих свой- ствах атмосферы на зондирующих длинах волн дает существенные искажения восстановленного озонового профиля [27]. В алгоритме расчета ВРО (1) аэрозольная коррекция учитывается в выражениях $C$ и $D$ введением реального распределения отношения рассеяния $R_{\text {off }}(H)$, в то время как в обычной «невозмущенной» атмосфере расчет ВРО можно проводить при значениях $R_{\text {off }}(H)=1$.

На СЛС проведен большой ряд измерений ВРО с учетом аэрозольной и температурной коррекцией. Для сопоставления спутниковых и лидарных данных важно учитывать вертикальное распределение их ошибок. Ошибка восстановления ВРО из лидарных измерений рассчитывается по следующей формуле:

$$
E_{\text {ums }}^{2}=e_{1}^{2}+e_{2}^{2}+e_{3}^{2},
$$

где $e_{1}$ - неточность сечения поглощения; $e_{2}-$ среднеквадратическая ошибка лидарного измерения в режиме счета фотонов; $e_{3}$ - погрешность восстановления отношения рассеяния. Для сечения поглощения взят квадрат ошибки его измерения, равный сумме квадратов систематической и предела статистической ошибки, раскрытые в работе [25], который соответствует квадрату числа $3,26 \%$.

Среднеквадратическая ошибка лидарного измерения в режиме счета фотонов:

$$
e_{2}^{2}=0,25\left[\frac{1}{N_{\text {on }}(H)}+\frac{1}{N_{\text {off }}(H)}\right] .
$$

Формула погрешности восстановления отношения рассеяния имеет вид

$$
\begin{gathered}
e_{3}^{2}=\frac{N_{\text {off }}(H)}{\left[N_{\text {off }}(H)-N_{\text {noise }}(H)\right]^{2}}+ \\
+\frac{N_{\text {off }}\left(H_{\text {calib }}\right)}{\left[N_{\text {off }}\left(H_{\text {calib }}\right)-N_{\text {noise }}\left(H_{\text {calib }}\right)\right]^{2}}+K,
\end{gathered}
$$

где $H_{\text {calib }}$ - высота калибровки; $N_{\text {noise }}$ - постоянная величина, $K$ - величина, появляющаяся вследствие предположений методики обработки и оцениваемая величина $3 \cdot(0,01)^{2}$.

В работе используются более актуальные коэффициенты поглощения с большим числом температурных точек по сравнению с ранними работами. Отличие применения нового и старого набора

\begin{tabular}{|c|c|c|c|c|c|c|c|c|c|c|c|}
\hline \multirow{2}{*}{$\begin{array}{l}\text { Длина волны, нм } \\
\text { Wavelength, nm }\end{array}$} & \multicolumn{11}{|c|}{ Температура/Temperature, $\mathrm{K}$} \\
\hline & 193 & 203 & 213 & 223 & 233 & 243 & 253 & 263 & 273 & 283 & 293 \\
\hline \multicolumn{12}{|c|}{ На линии поглощения/Online } \\
\hline 299 & $4,12 \cdot 10^{-19}$ & $4,15 \cdot 10^{-19}$ & $4,25 \cdot 10^{-19}$ & $4,15 \cdot 10^{-19}$ & $4,3 \cdot 10^{-19}$ & $4,25 \cdot 10^{-19}$ & $4,36 \cdot 10^{-19}$ & $4,36 \cdot 10^{-19}$ & $4,38 \cdot 10^{-19}$ & $4,46 \cdot 10^{-19}$ & $4,58 \cdot 10^{-19}$ \\
\hline 308 & $1,13 \cdot 10^{-19}$ & $1,14 \cdot 10^{-19}$ & $1,16 \cdot 10^{-19}$ & $1,17 \cdot 10^{-19}$ & $1,18 \cdot 10^{-19}$ & $1,19 \cdot 10^{-19}$ & $1,24 \cdot 10^{-19}$ & $1,25 \cdot 10^{-19}$ & $1,28 \cdot 10^{-19}$ & $1,31 \cdot 10^{-19}$ & $1,35 \cdot 10^{-19}$ \\
\hline \multicolumn{12}{|c|}{ Вне линии поглощения/Offline } \\
\hline 341 & $5,6210^{-22}$ & $5,94 \quad 10^{-22}$ & $6,110^{-22}$ & $6,9510^{-22}$ & $7,0510^{-22}$ & 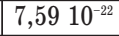 & $8,1510^{-22}$ & $8,910^{-22}$ & $9,910^{-22}$ & $1,08 \quad 10^{-21}$ & $1,1510^{-21}$ \\
\hline 353 & $4,95 \cdot 10^{-23}$ & $6,4 \cdot 10^{-23}$ & $7,25 \cdot 10^{-23}$ & $8,88 \cdot 10^{-23}$ & $9,57 \cdot 10^{-23}$ & $1,1 \cdot 10^{-22}$ & $1,27 \cdot 10^{-22}$ & $1,45 \cdot 10^{-22}$ & $1,67 \cdot 10^{-22}$ & $2,02 \cdot 10^{-22}$ & $2,38 \cdot 10^{-22}$ \\
\hline
\end{tabular}
сечения поглощения представлено в виде восстановленных профилей на рис. 2.

Таблица 2. Сечения поглощения озона (c.⿲丿丶丶 2 для диапазона 193-293 К на длинах волн зондирования озона [25, 26 ]

Table 2. Ozone absorption cross sections $\left(\mathrm{cm}^{2}\right)$ for the range of 193-293 $\mathrm{K}$ at the wavelengths of ozone sounding [25, 26] 

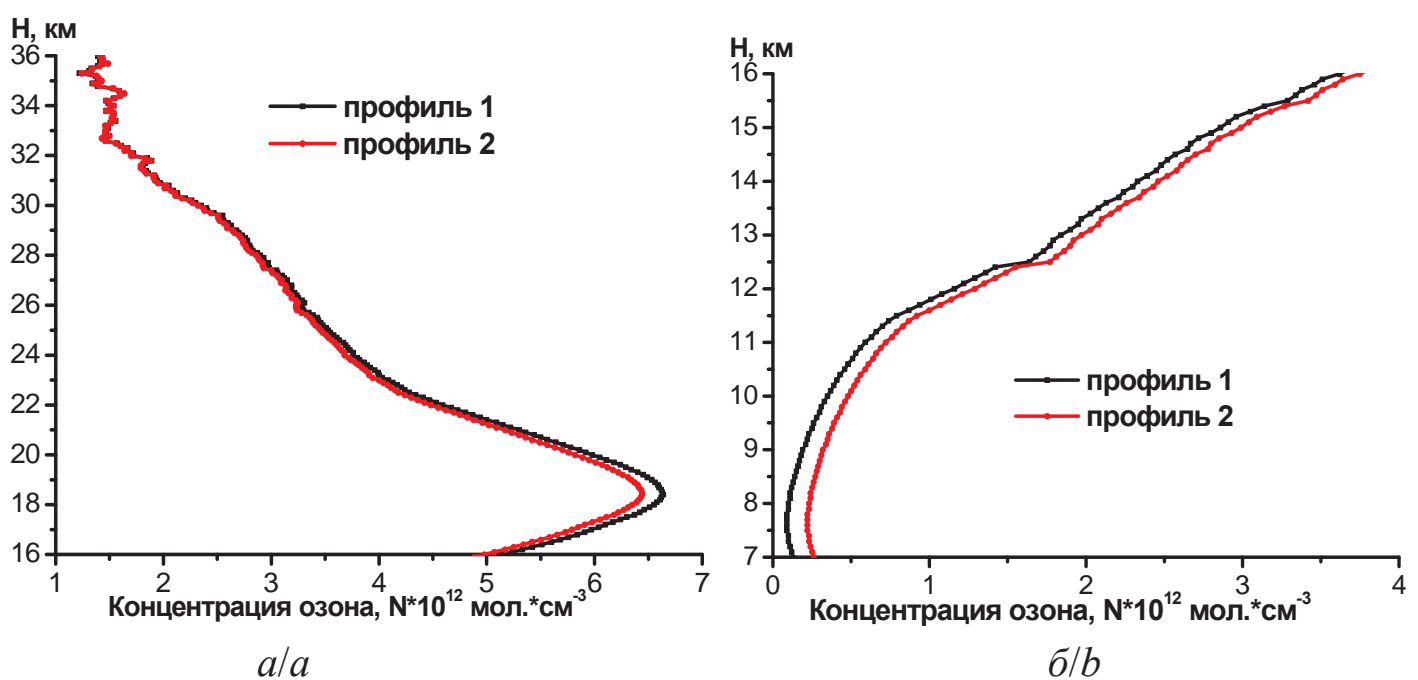

Рис. 2. Сопоставление профилей озона: а) ВРО стратосферы за 10.03.2017 г.; б) ВРО верхней тропосферы - нижней стратосферы за 21.12.2017 2.

Fig. 2. Comparison of ozone profiles: a) stratosphere OVD for 10.03.2017; b) upper troposphere - lower stratosphere OVD for 21.12.2017

Профиль 1 на рис. 2 соответствует восстановленному профилю озона с использованием новых коэффициентов поглощения по работам [25, 26], профиль 2 относится к более ранним работам $[28,29]$.

\section{Результаты сопоставления лидарных профилей со спутниковыми данными}

За январь 2018 г. на станции лидарного зондирования ИОА СО РАН получили 18 измерений стратосферного ВРО, также получили 18 измерений верхней тропосферы - нижней стратосферы во второй половине года. Наиболее удачные 14 измерений представлены в этой работе. Используя представленную выше методику, рассчитывались восстановленные профили озона стратосферы и верхней тропосферы - нижней стратосферы. Они сопоставляются с некоторыми профилями, полученными с помощью интерферометра IASI, установленного на борту метеорологического спутника MetOpt, а также с микроволновым радиометром MLS, установленного на борту метеорологического спутника AURA. Координаты и время лидарного и спутникового зондирования приведены в табл. 3,4 . Лидарные измерения озона над городом Томск проводились в вечернее и ночное время в условиях безоблачного неба.

Все профили ВРО приведены в сравнении с моделью Крюгера [30]. Стратосферные измерения озона представлены в виде трех кривых: восстановленный лидарный профиль, AURA профиль и модель Крюгера. Из рис. 3, а видно, что профили, восстановленные из лидарных и спутниковых данных, совпадают с небольшими отклонениями на протяжении всего слоя измерений. Отклонения обусловлены разницей между пространственным разрешением лидара $(100 \mathrm{~m})$ и спутника AURA (1,3 км и больше), соответственно количество точек, формирующих ВРО, больше у лидара, что так- же влияет на поведение кривой восстановленного BPO.

Измерения приведены к общему высотному диапазону 16-38 км для более удобного сравнения всех профилей. Из рис. $3, a$ видно, что для всего периода наблюдений прослеживается в ряде измерений завышение концентраций озона AURA по сравнению с данными лидара в диапазоне от 22 до $38 \mathrm{kM}$.

Из анализа натурных данных стратосферного ВРО за весь рассматриваемый период 2018 г., которые представлены на рис. 3, можно сделать следующие выводы. Незначительная разница между профилями концентраций (рис. $3, a, \sigma)$, полученными на лидаре и AURA, присутствует в начале и на хвосте высотной шкалы. Вследствие этого средняя разность (Лидар-AURA), или отклонение, по всем дням измерений в концентрациях озона изменяется от $-0,54 \cdot 10^{12}$ мол. $\mathrm{cm}^{-3}$ на высоте 24,6 км до $0,14 \cdot 10^{12}$ мол. см $^{-3}$ на 17,5 км (рис. 3, б). Максимальное по всем профилям отклонение составляет от $-0,33 \cdot 10^{12}$ мол. см $^{-3}$ на высоте $26,3 \mathrm{\kappa м}$ до $1,54 \cdot 10^{12}$ мол. см $^{-3}$ на 16 км. Минимальное отклонение также по всем профилям изменяется от $-1,68 \cdot 10^{12}$ мол. $\mathrm{cm}^{-3}$ на $16 \mathrm{kм}$ до $-0,36 \cdot 10^{12}$ мол. $\mathrm{CM}^{-3}$ на высоте 35 км.

Поскольку озон имеет заметный ход, то для того, чтобы найти относительные погрешности его измерения прибором MLS (AURA), разность концентраций нормировали на значения, полученные лидаром: 100*(Лидар - AURA)/Лидар. Эти данные представлены на рис. 3 , в, из которого видно, что средняя относительная разность положительна в диапазоне высот 16,5...19,2 км, где достигает $2,35 \%$ на 17,9 км.

Средняя относительная разность отрицательна в диапазоне высот $16 \ldots 16,4$ и 19,3...38 км, где достигает максимального отрицательного значения $-87,93 \%$ на 38 км. Максимальная относи- 
тельная разность изменяется от $-11,86$ до $26,74 \%$ на высоте 26,3 и 36,3 км соответственно. Минимальная по всем профилям относительная разность в этих высотах составляет $-172,54 \%$ на 37,6 км и достигает $-10,49 \%$ на 19 км. На высоте 16 км относительная разница варьируется в диапазоне минимума и максимума $-45,17 . .23,91 \%$, на 38 км от $-125,56$ до $15,72 \%$.

Tаблица 3. Координаты и время спутникового AURA (MLS) и лидарного зондирования, использованные для сравне ния ВРО за январь 2018 2.

Table 3. Coordinates and time of satellite AURA (MLS) and lidar sounding, used to compare the OVD in January 2018

\begin{tabular}{|c|c|c|c|c|}
\hline \multirow[b]{2}{*}{$\begin{array}{l}\text { Дата } \\
\text { Date }\end{array}$} & \multicolumn{2}{|c|}{$\begin{array}{l}\text { Лидарная станция } \\
\text { Lidar station }\end{array}$} & \multicolumn{2}{|c|}{$\begin{array}{l}\text { Спутник AURA (MLS) } \\
\text { Satellite AURA (MLS) }\end{array}$} \\
\hline & $\begin{array}{c}\text { Время по } \\
\text { Гринвичу } \\
\text { GTM }\end{array}$ & \begin{tabular}{|c|} 
Координаты \\
Coordinate \\
(56,5 с.ш.; \\
85,0 в.д.)
\end{tabular} & $\begin{array}{c}\text { Время по } \\
\text { Гринвичу } \\
\text { GTM }\end{array}$ & $\begin{array}{l}\text { Koopдинаты } \\
\text { Coordinate }\end{array}$ \\
\hline 13.01 .18 & \multicolumn{2}{|c|}{$11: 55-12: 25$} & 07:07 & 60.43 с.ш. 84.56 в.д \\
\hline 13.01 .18 & \multicolumn{2}{|c|}{$12: 25-13: 04$} & 07:07 & 60.43 с.ш. 84.56 в.д \\
\hline 22.01 .18 & \multicolumn{2}{|c|}{$11: 42-12: 12$} & 07:01 & 60.43 с.ш. 86.10 в.д \\
\hline 22.01 .18 & \multicolumn{2}{|c|}{$12: 12-12: 42$} & 07:01 & 60.43 с.ш. 86.10 в.д \\
\hline 23.01 .18 & \multicolumn{2}{|c|}{$13: 13-13: 43$} & $21: 14$ & 51.74 с.ш. 78.10 в.д \\
\hline 23.01 .18 & \multicolumn{2}{|c|}{$13: 43-14: 13$} & $21: 14$ & 51.74 с.ш. 78.10 в.д \\
\hline 24.01 .18 & \multicolumn{2}{|c|}{ 11:42-12:12 } & $06: 49$ & 60.43 с.ш. 89.20 в.д \\
\hline 24.01 .18 & \multicolumn{2}{|c|}{$12: 12-12: 42$} & $06: 49$ & 60.43 с.ш. 89.20 в.д \\
\hline 26.01 .18 & \multicolumn{2}{|c|}{$13: 19-13: 49$} & $06: 36$ & 60.43 с.ш. 92.29 в.д \\
\hline 26.01 .18 & \multicolumn{2}{|c|}{$13: 49-13: 49$} & $06: 36$ & 60.43 с.ш. 92.29 в.д \\
\hline 30.01 .18 & \multicolumn{2}{|c|}{$12: 15-12: 45$} & $07: 49$ & 54.65 с.ш. 77.13 в.д \\
\hline 30.01 .18 & \multicolumn{2}{|c|}{$12: 45-13: 15$} & $07: 49$ & 54.65 с.ш. 77.13 в.д \\
\hline 31.01 .18 & \multicolumn{2}{|c|}{$13: 15-13: 45$} & $06: 55$ & 58.99 с.ш. 88.61 в.д \\
\hline 31.01 .18 & \multicolumn{2}{|c|}{$13: 45-14: 15$} & $06: 55$ & 58.99 с.ш. 88.61 в.д \\
\hline
\end{tabular}

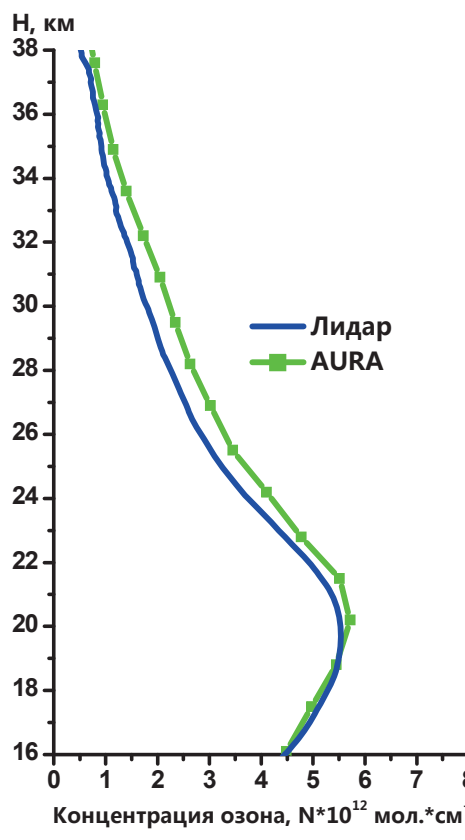

$a / a$

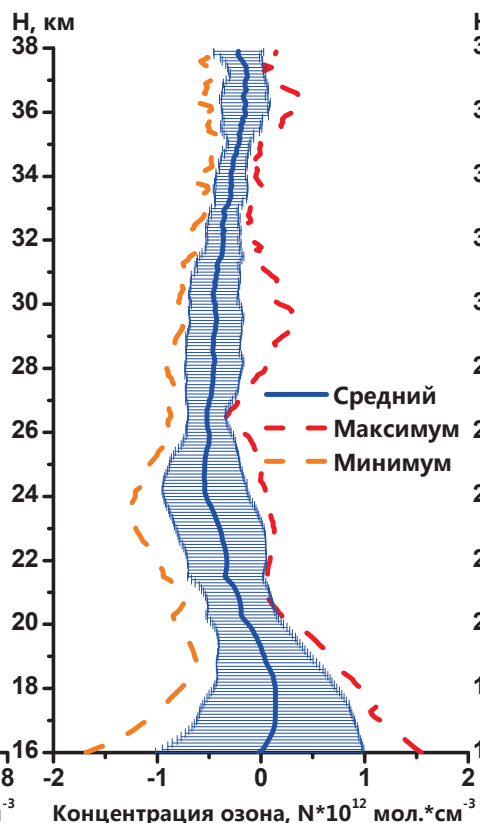

$\sigma / b$
Таблица 4. Координаты и время спутникового MetOp (IASI) u лидарного зондирования тропосферы, использованные для сравнения ВРО за январь 2018 2.

Table 4. Coordinates and time of satellite MetOp (IASI) and lidar sounding, used to compare the OVD in January 2018

\begin{tabular}{|c|c|c|c|c|}
\hline \multirow[b]{2}{*}{$\begin{array}{l}\text { Дата } \\
\text { Date }\end{array}$} & \multicolumn{2}{|c|}{$\begin{array}{l}\text { Лидарная станция } \\
\text { Lidar station }\end{array}$} & \multicolumn{2}{|c|}{$\begin{array}{l}\text { Спутник Met0p (IASI) } \\
\text { Satellite Met0p (IASI) }\end{array}$} \\
\hline & $\begin{array}{c}\text { Время по } \\
\text { Гринвичу } \\
\text { GTM }\end{array}$ & $\begin{array}{c}\text { Координаты } \\
\text { Coordinate } \\
\text { (56,5 с.ш.; } \\
\text { 85,0 в.д.) }\end{array}$ & $\begin{array}{c}\text { Время по } \\
\text { Гринвичу } \\
\text { GTM }\end{array}$ & $\begin{array}{l}\text { Коoрдинаты } \\
\text { Coordinate }\end{array}$ \\
\hline 13.01 .18 & \multicolumn{2}{|c|}{ 13:28-14:02 } & $13: 53$ & 56.47 с.ш. 85.04 в.д \\
\hline 13.01 .18 & \multicolumn{2}{|c|}{$14: 03-14: 37$} & $13: 53$ & 56.47 с.ш. 85.04 в.Д \\
\hline 22.01 .18 & \multicolumn{2}{|c|}{$12: 58-13: 32$} & 14:08 & 56.47 с.ш. 85.04 в.д \\
\hline 22.01 .18 & \multicolumn{2}{|c|}{$13: 32-14: 06$} & 14:08 & 56.47 с.ш. 85.04 в.д \\
\hline 23.01 .18 & \multicolumn{2}{|c|}{$11: 41-12: 15$} & $14: 29$ & 56.47 с.ш. 85.04 в.д \\
\hline 23.01 .18 & \multicolumn{2}{|c|}{$12: 15-12: 49$} & $14: 29$ & 56.47 с.ш. 85.04 в.д \\
\hline 24.01 .18 & \multicolumn{2}{|c|}{$13: 04-13: 38$} & $14: 08$ & 56.47 с.ш. 85.04 в.д \\
\hline 24.01 .18 & \multicolumn{2}{|c|}{$13: 38-14: 12$} & $14: 08$ & 56.47 с.ш. 85.04 в.д \\
\hline 26.01 .18 & \multicolumn{2}{|c|}{$11: 51-12: 25$} & $14: 23$ & 56.47 с.ш. 85.04 в.д \\
\hline 26.01 .18 & \multicolumn{2}{|c|}{$12: 25-12: 59$} & $14: 23$ & 56.47 с.ш. 85.04 в.д \\
\hline 30.01 .18 & \multicolumn{2}{|c|}{$13: 27-14: 01$} & $13: 44$ & 56.47 с.ш. 85.04 в.д \\
\hline 30.01 .18 & \multicolumn{2}{|c|}{ 14:01-14:35 } & $13: 44$ & 56.47 с.ш. 85.04 в.д \\
\hline 31.01 .18 & \multirow{2}{*}{\multicolumn{2}{|c|}{$\begin{array}{l}11: 52-12: 26 \\
12: 26-13: 00\end{array}$}} & $14: 20$ & 56.47 с.ш. 85.04 в.д \\
\hline 31.01 .18 & & & $14: 20$ & 56.47 с.ш. 85.04 в.д \\
\hline
\end{tabular}

Таким образом, проведенное сопоставление показывает, что абсолютные различия концентрации озона, измеренные на лидаре и прибором MLS (AURA), могут изменяться от $-1,68 \cdot 10^{12}$ мол. $\mathrm{cm}^{-3}$ до $1,54 \cdot 10^{12}$ мол. см $^{-3}$, и тогда, учитывая минимум и максимум относительной разницы, получаем ее варьирование, которое находится в диапазоне $-125,56 \ldots+26,74 \%$.

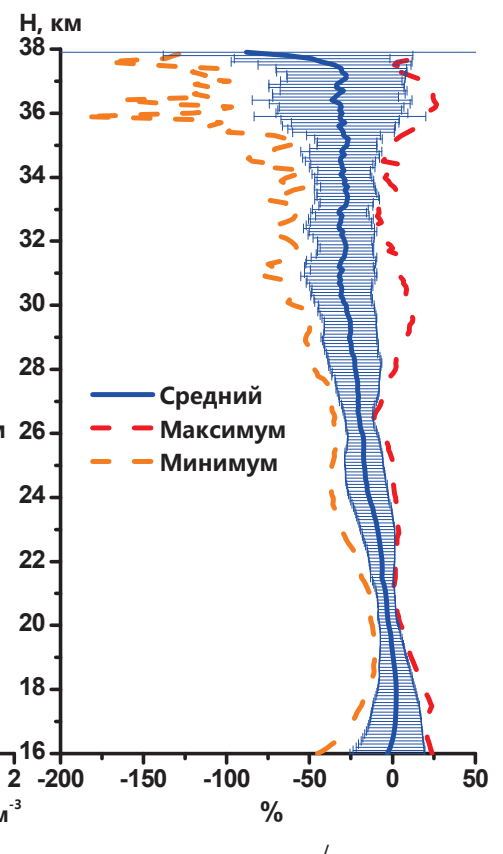

Puс. 3. Средние вертикальные профили озона и их различия: а) средние профили; б) различия Лидар и AURA в абсолютных единицах; в) относительные различия 100*(Лидар-AURA)/Лидар

Fig. 3. Average vertical profiles of ozone and their differences: a) average profiles; $b$ ) differences between Lidar and AURA in absolute units; c) relative differences 100*(Lidar-AURA)/Lidar 
На рис. 4 представлено вертикальное распределение средней ошибки восстановления и, соответственно, средние профили озона лидарных и спутниковых измерений стратосферы. При восстановлении стратосферного озона из лидарных данных считаем, что атмосфера находится в невозмущенном состоянии, поэтому в расчете ошибок ВРО аэрозольная коррекция не участвует и соответственно неточности отношения рассеяния не учитываются.

Лидарные профили озона, полученные на СЛС и BPO по IASI данным (рис. 5), приведены к общему высотному диапазону $6-18$ км для более удобного сравнения всех профилей.

Из рис. $5, a$ видно, что для всего периода наблюдений прослеживается в ряде измерений завышение концентраций озона по лидару в сравнении с данными IASI в диапазоне от 12 до 18 км.

Из анализа данных ВРО верхней тропосферы нижней стратосферы за весь рассматриваемый период 2018 г., приведенных на рис. 5, можно сделать следующие выводы. Разница между профилями концентраций озона (рис. $5, a$, б), полученная на лидаре и IASI, растет с увеличением высоты. Вследствие этого средняя разность (Лидар-IASI) или отклонение по всем дням измерений в концентрациях озона изменяются от $0,04 \cdot 10^{12}$ мол. $\mathrm{cm}^{-3}$ на высоте 6,7 км до $1,69 \cdot 10^{12}$ мол. см $^{-3}$ на 16,3 км (рис. 5, б). Максимальное по всем профилям отклонение составляет от $0,47 \cdot 10^{12}$ мол. см $^{-3}$ на высоте 6,5 км до $2,35 \cdot 10^{12}$ мол. $\mathrm{cm}^{-3}$ на 16,1 км. Минимальное отклонение также по всем профилям изменяется от $-0,36 \cdot 10^{12}$ мол. с ${ }^{-3}$ на $6,4 \mathrm{\kappa м}$ до $0,99 \cdot 10^{12}$ мол. см $^{-3}$ на высоте 16,8 км.
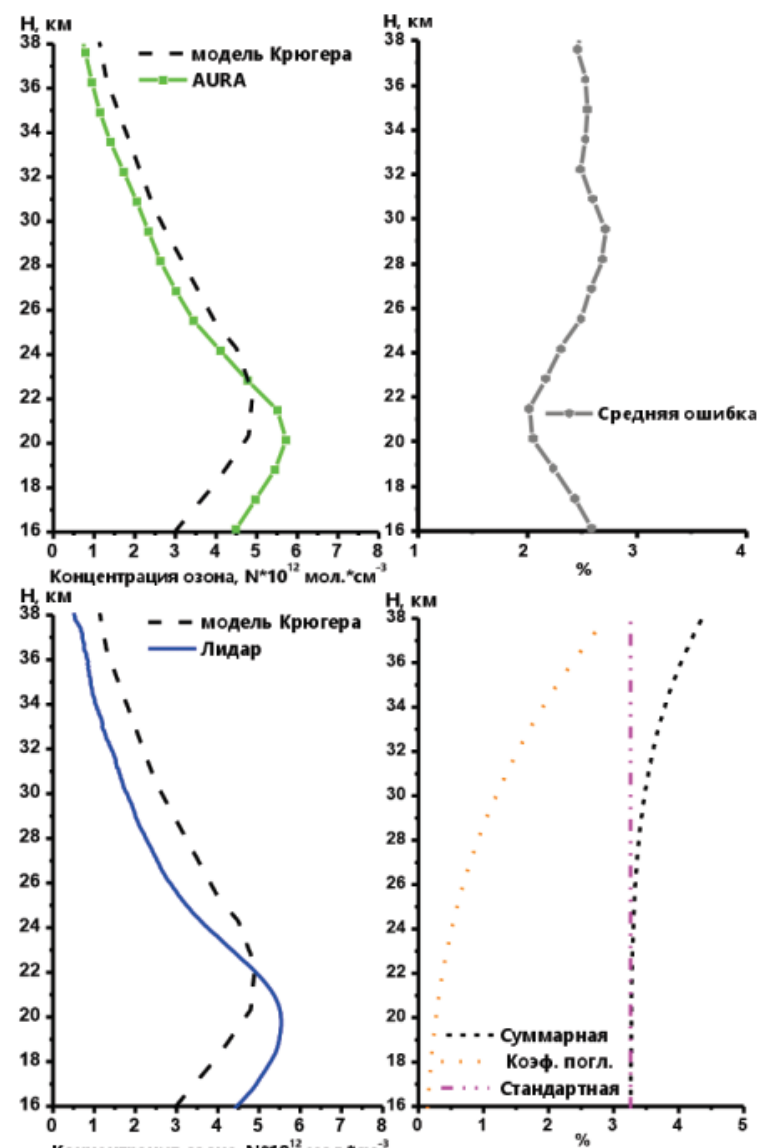

Puc. 4. Средние BPO лидара и AURA с ошибками восстановления

Fig. 4. Average OVD for LIDAR and AURA with recovery errors

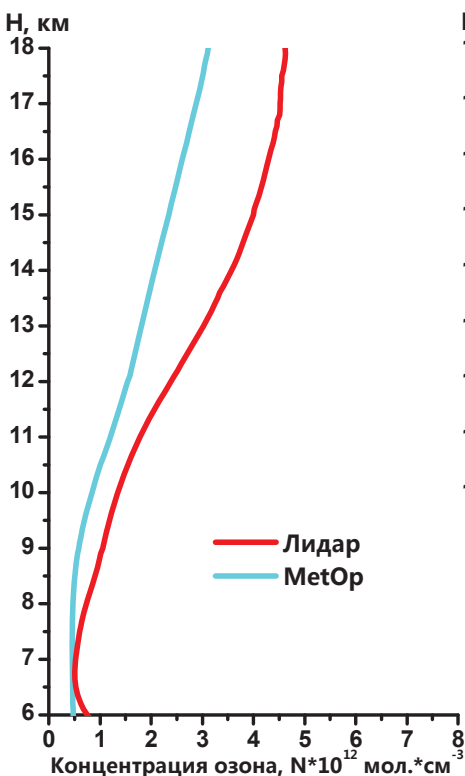

$a / a$

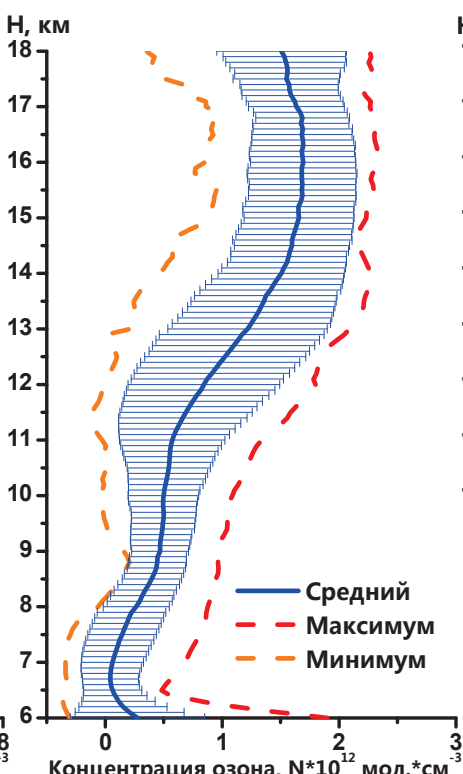

$\sigma / b$

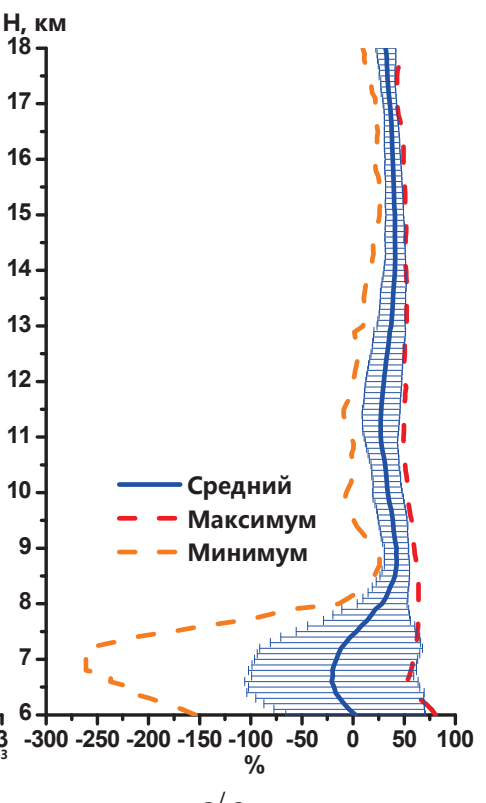

$B / c$

Pис. 5. Средние профили озона и их различия: а) средние профили; б) различия Лидара и IASI (MetOp) в абсолютных единицах; в) относительные различия 100*( Лидар-IASI)/Лидар

Fig. 5. Average profiles of ozone and their differences: a) average profiles; b) differences between Lidar and IASI (MetOp) in absolute units; c) relative differences 100*(Lidar-IASI)/Lidar 
Поскольку озон имеет заметный годовой ход, то для того, чтобы найти относительные погрешности его измерения прибором IASI (MetOp), разность концентраций нормировали на значения, полученные лидаром: 100*(Лидар - IASI)/ Лидар. Эти данные представлены на рис. 5 в, из которого видно, что средняя относительная разность положительна в диапазоне высот 6 и 7,5...18 км, где достигает $42,75 \%$ на 8,7 км, и отрицательна в диапазоне высот $6,1 \ldots 7,4$ км, где достигает - $20,98 \%$ на 6,6 км.

Максимальная относительная разность изменяется от 42,49 до $80,73 \%$ на высоте 17,3 и 6 км соответственно. Минимальная по всем профилям относительная разность в этих высотах составляет $-261,53 \%$ на 6,8 км и достигает $26,88 \%$ на 8,9 км. Такое значение минимума относительной разности обусловлено измерениями (13.01.18, 22.01.18 и др.), когда значения спутникового профиля значительно больше лидарного. Поэтому получается такая петля в высотах от 6 до 8 км на рис. 5 , в. На высоте 18 км относительная разница варьируется в диапазоне минимума и максимума от 9,67 до $44,99 \%$, на 6 км от $-154,16$ до $80,73 \%$.

Таким образом, проведенное сопоставление показывает, что абсолютные различия концентрации озона, измеренные на лидаре и прибором IASI (MetOp), могут изменяться от $-0,36 \cdot 10^{12}$ мол. $\mathrm{cm}^{-3}$ до $2,35 \cdot 10^{12}$ мол. см $^{-3}$ и тогда, учитывая минимум и максимум относительной разницы, получаем ее варьирование, которое находится в диапазоне $-261,53 \ldots+80,73 \%$.

Из рис. 6 видно, что ошибка восстановления от $\sim 6,7 \%$ на высоте 5 км до $12 \%$ нм 18 км. Видно, что существенный вклад в общую ошибку вносит неточность отношения рассеяния.
На рис. 7 приведены некоторые общие ВРО, которые объединяют лидарный контроль стратосферы и верхней тропосферы - нижней стратосферы в общую кривую ВРО. Такой комплексный подход измерений ВРО дает возможность более полно производить лидарный контроль процессов стратосферно-тропосферного обмена воздушных масс. Из рис. 7 видно, что стратосферные лидарные и спутниковые наблюдения хорошо согласуются.

\section{Заключение}

Таким образом, создан лидар для измерения ВРО в верхней тропосфере - стратосфере в высотном диапазоне $\sim(5-45)$ км на длинах волн $299 / 341$ и 308/353 нм. Следующим шагом модернизации лидарного комплекса для измерения ВРО является модификация лидарного приемника и настройка передатчиков для одновременного зондирования всей тропосферы и стратосферы или создание еще одного лидарного приемника, настроенного на прием сигналов второй пары длин волн. Это позволит сократить время измерений вдвое и соответственно увеличить количество измерений. Последующим шагом стоит реализация зондирования неохваченных высот 0,1-5 км, что позволит контролировать всю озоносферу.

Результаты сопоставления восстановленных лидарных профилей ВРО в верхней тропосфере нижней стратосфере и стратосфере со спутниковыми профилями AURA (MLS) и IASI/MetOp, a также сшитый профиль ВРО верхней тропосферы - стратосферы в сравнении со среднеширотной моделью Крюгера подтверждают перспективность использования пар длин волн зондирования озона 299/341 и $308 / 353$ нм. Стоит отметить, что

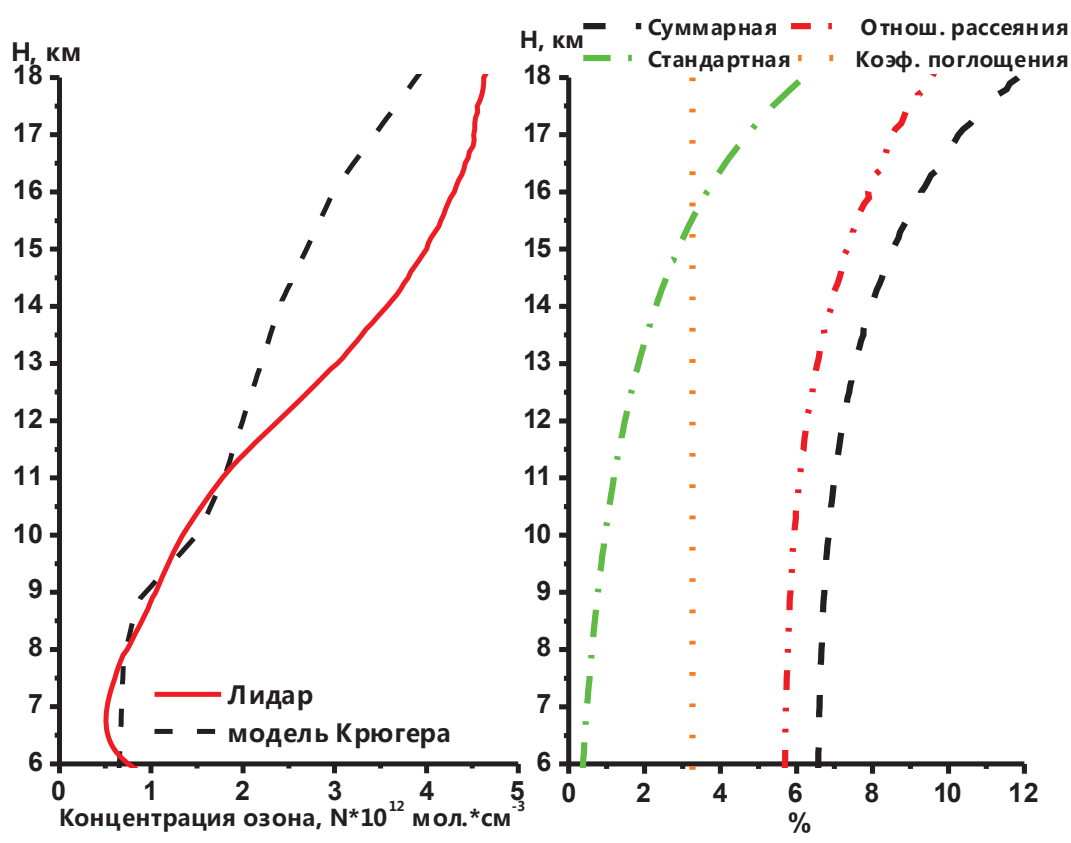

Pис. 6. Средние ВРО лидара и ошибки восстановления в верхней тропосфере - нижней стратосфере

Fig. 6. Average OVD for LIDAR and recovery errors in upper troposphere - lower stratosphere 

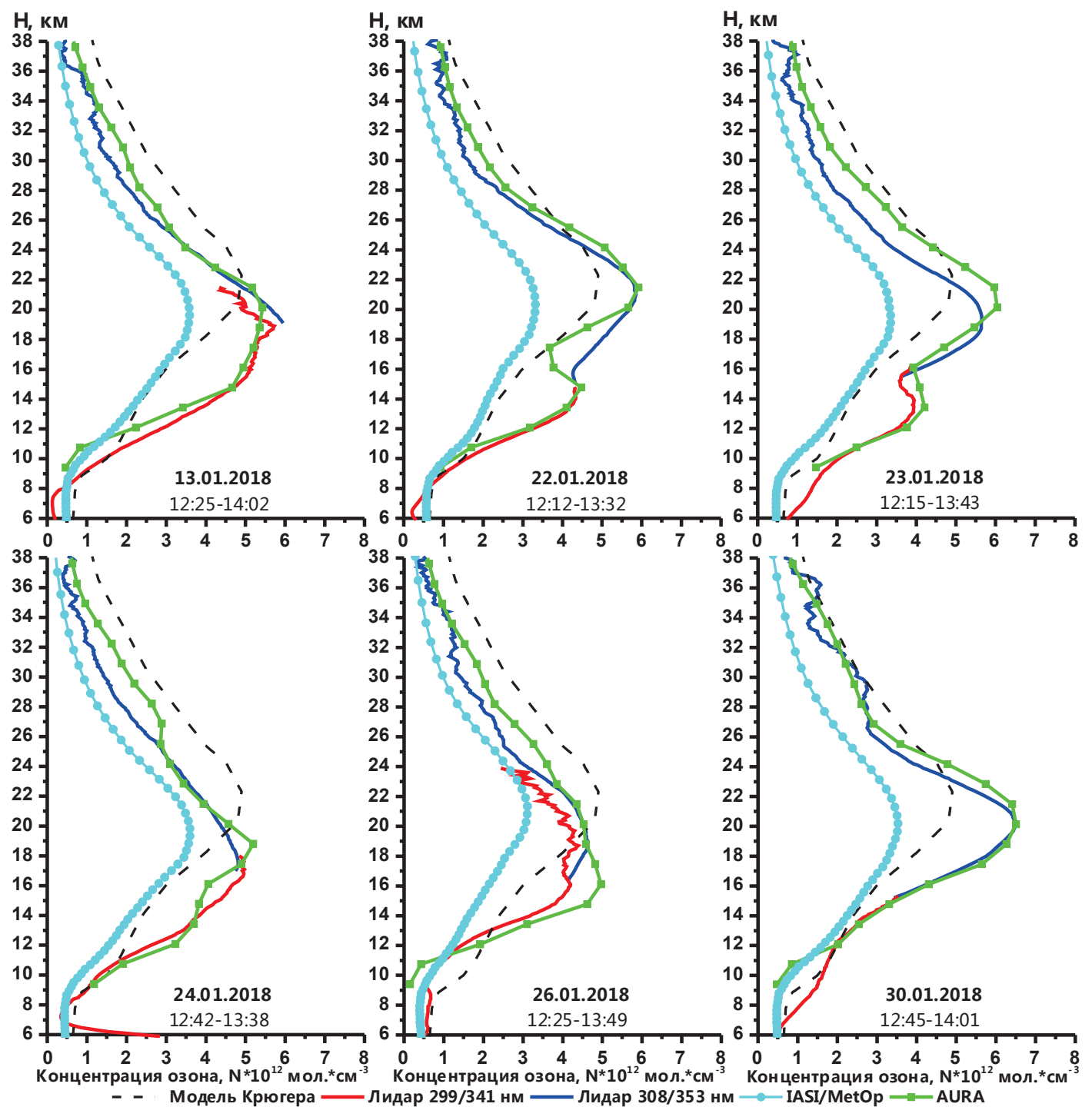

Puc.7. Сопоставление вертикальных профилей озона верхней тропосферы - стратосферы с спутниковыми данными AURA и MetOp

Fig. 7. Comparison of vertical ozone profiles of the upper troposphere - stratosphere with satellite data AURA and MetOp

восстановленные профили высотного распределения концентрации озона больше тяготеют к профилям спутниковых данных AURA, чем к модели Крюгера.

\section{СПИСОК ЛИТЕРАТУРЫ}

1. Лидарное зондирование озона в верхней тропосфере - нижней стратосфере: методика и результаты измерений / В.Д. Бурлаков, С.И. Долгий, А.А. Невзоров, А.В. Невзоров, О.А. Романовский, О.В. Харченко // Известия Томского политехнического университета. Инжиниринг георесурсов. - 2015. T. 326. - № 9. - C. 124-132.

2. Weitkamp C. Lidar: range resolved optical remote sensing of the atmosphere. - New York: Springer-Verlag New York, 2005. $456 \mathrm{p}$.

3. Лидарный спектроскопический газоанализ атмосферы С.М. Бобровников, Г.Г. Матвиенко, О.А. Романовский, И.Б. Сериков, А.Я. Суханов. - Томск: Изд-во ИОА СО РАН, 2014. $-510 \mathrm{c}$.
Работа выполнена при частичной финансовой поддержке стипендии Президента РФ по поддержке молодых ученых и аспирантов (СП3926.2018.3).

4. Лидарный мониторинг облачных и аэрозольных полей, малых газовых составляющих и метеопараметров атмосферы / Ю.С. Балин, А.Г. Боровой, В.Д. Бурлаков, С.И. Долгий, М.Г. Клемашева, А.В. Коношонкин, Г.П. Коханенко, Н.В. Кустова, В.Н. Маричев, Г.Г. Матвиенко, А.А. Невзоров, А.В. Невзоров, И.Э. Пеннер, О.А. Романовский, С.В. Самойлова, А.Я. Суханов, О.В. Харченко, В.А. Шишко. - Томск: Изд-во ИОА СО РАН, 2015. $-450 \mathrm{c}$.

5. Сибирская лидарная станция: аппаратура и результаты / Г.Г. Матвиенко, Ю.С. Балин, С.М. Бобровников, О.А. Романовский, Г.П. Коханенко, С.В. Самойлова, И. Э. Пеннер, Е.В. Горлов, В.И. Жарков, С.А. Садовников, О.В. Харченко, С.В. Яковлев, О.Е. Баженов, В.Д. Бурлаков, С.И. Долгий, А.П. Макеев, А.А. Невзоров, А.В. Невзоров. - Томск: Изд-во ИОА СО РАН, 2016. - 440 c. 
6. Comparison of ozone profiles from DIAL, MLS, and chemical transport model simulations over Río Gallegos, Argentina, during the spring Antarctic vortex breakup, 2009 / T. Sugita, H. Akiyoshi, E. Wolfram, J. Salvador, H. Ohyama, A. Mizuno // Atmospheric Measurement Techniques. - 2017. - V. 10. Iss. 12. - P. 4947-4964.

7. Tropospheric ozone profiles by DIAL at Maïdo Observatory (Reunion Island): system description, instrumental performance and result comparison with ozone external data set / V. Duflot, J.-L. Baray, G. Payen, N. Marquestaut, Fr. Posny, J.-M. Metzger, B. Langerock, C. Vigouroux, J. Hadji-Lazaro, Th. Portafaix, M. De Mazière, P.-Fr. Coheur, C. Clerbaux, J.-P. Cammas // Atmospheric Measurement Techniques. - 2017. - V. 10. Iss. 9. - P. 3359-3373.

8. Airborne lidar measurements of aerosol and ozone above the Canadian oil sands region / M. Aggarwal, J. Whiteway, J. Seabrook, L. Gray, K. Strawbridge, P. Liu, J. O'Brien, Sh.-M. Li, R. McLaren // Atmospheric Measurement Techniques. - 2018. - V. 11. Iss. 6. - P. 3829-3849.

9. Quantifying TOLNet ozone lidar accuracy during the 2014 DISCOVER-AQ and FRAPPÉ campaigns / L. Wang, M.J. Newchurch, R.J. Alvarez II, T.A. Berkoff, S. Brown, W. Carrion, R.J. De Young, B.J. Johnson, R. Ganoe, G. Gronoff, G. Kirgis, Sh. Kuang, A.0. Langford, T. Leblanc, E.E. McDuffie, T.J. McGee, D. Pliutau, C.J. Senff, J.T. Sullivan, G. Sumnicht, L.W. Twigg, A.J. Weinheimer // Atmospheric Measurement Techniques. - 2017. - V. 10. - Iss. 10. - P. 3865-3876.

10. Algorithm improvement and validation of National Institute for Environmental Studies ozone differential absorption lidar at the Tsukuba Network for Detection of Stratospheric Change complementary station / Ch.B. Park, H. Nakane, N. Sugimoto, Ich. Matsui, Y. Sasano, Y. Fujinuma, Iz. Ikeuchi, J.-Ich. Kurokawa, N. Furuhashi // Applied Optics. - 2006. - V. 45. - № 15. P. 3561-3576.

11. Tropospheric ozone differential-absorption lidar using stimulated Raman scattering in carbon dioxide/ M. Nakazato, T. Nagai, T. Sakai, Y. Hirose // Applied 0ptics. - 2007. - V. 46. - № 12. P. 2269-2279.

12. Godin-Beekmanna S., Songa T., Heeseb B. Long-term DIAL monitoring of the stratosphericozone vertical distribution // Proceedings of SPIE. - 2003. - V. 4893. - P. 251-263.

13. Gaudel A., Ancellet G., Godin-Beekmann S. Analysis of 20 years of tropospheric ozone vertical profiles by lidar and ECC at Observatoire de Haute Provence (OHP) at 44N, 6.7E // Atmospheric Environment. - 2015. - V. 113. - P. 78-89.

14. McDermid I.S., Godin S.M., Lindquist L.0. Ground-based laser DIAL system for long-term measurements of stratospheric ozone // Applied Optics. - 1990. - V. 29. - № 25. - P. 3603-3612.

15. Redesign and improved performance of the tropospheric ozone lidar at the Jet Propulsion Laboratory Table Mountain Facility / I.S. McDermid, G. Beyerle, D.A. Haner, T. Leblanc // Applied 0ptics. - 2002. - V. 41. - № 36. - P. 7550-7555.

16. Intercomparison of stratospheric ozone and temperature profiles during the October 2005 Hohenpeißenberg Ozone Profiling Experiment (HOPE) / W. Steinbrecht, T.J. McGee, L.W. Twigg, H. Claude, F. Sch"onenborn, G.K. Sumnicht, D. Silbert // Atmospheric Measurement Techniques. - 2009. - V. 2. - Iss. 1. P. $125-145$.
17. A mobile differential absorption lidar to measure sub-hourly fluctuation of tropospheric ozone profiles in the Baltimore-Washington, D.C. region / J.T. Sullivan, T.J. McGee, G.K. Sumnicht, L.W. Twigg, R.M. Hoff // Atmospheric Measurement Techniques. - 2014. - V. 7. - Iss. 10. - P. 3529-3548.

18. Lidar Measurements of Variability of the Vertical Ozone Distribution Caused by the Stratosphere-Troposphere Exchange in the Far East Region / A.N. Pavlov, S.Yu. Stolyarchuk, K.A. Shmirko, 0.A. Bukin // Atmospheric and Oceanic Optics. - 2013. V. 26. - № 2. - P. 126-134.

19. Бурлаков В.Д., Долгий С.И., Невзоров А.В. Модернизация измерительного комплекса Сибирской лидарной станции // Оптика атмосферы и океана. - 2004. - Т. 5. - № 10. C. $1022-1027$.

20. Intercomparison of ozone vertical profile measurements by differential absorption lidar and IASI/Met0p satellite in the upper troposphere-lower stratosphere / S.I. Dolgii, A.A. Nevzorov, A.V. Nevzorov, 0.A. Romanovskii, 0.V. Kharchenko // Remote Sensing. - 2017. - V. 9. - № 5. - P. 447-462.

21. MLS data documentation. URL: https://mls.jpl.nasa.gov/data/datadocs.php (дата обращения 30.07.18).

22. Goddard Space Flight Center. Данные бортового прибора MLS спутника AURA. URL: https://avdc.gsfc.nasa.gov/pub/data/satellite/Aura/MLS/V04/L2GPOVP/03/ (дата обращения 30.07.18).

23. IASI on Metop-A: Operational Level 2 retrievals after five years in orbit / T. August, D. Klaes, P. Schlüssel, T. Hultberg, M. Crapeau, A. Arriaga, A. 0'Carroll, D. Coppens, R. Munro, X. Calbet // Journal of Quantitative Spectroscopy and Radiative Transfer. - 2012. - V. 113. - № 11. - P. 1340-1371.

24. Mezheris R. Laser remote sensing. - Moscow: World Publ., 1987. - $552 \mathrm{P}$.

25. High spectral resolution ozone absorption cross-sections - P. 1: Measurements, data analysis and comparison with previous measurements around $293 \mathrm{~K}$ / V. Gorshelev, A. Serdyuchenko, M. Weber, W. Chehade, J.P. Burrows // Atmospheric Measurement Techniques. - 2014. - V. 7. - Iss. 2. - P. 609-624.

26. High spectral resolution ozone absorption cross-sections - Part 2: Temperature dependence / A. Serdyuchenko, V. Gorshelev, M. Weber, W. Chehade, J.P. Burrows // Atmospheric Measurement Techniques. - 2014. - V. 7. - Iss. 2. - P. 625-636.

27. El'nikov A.V., Zuev V.V. Bifrequency laser sounding of stratospheric ozone under conditions of high degree of aerosol loading // Atmospheric and oceanic optics. - 1992. - V. 5. - № 10. P. 681-683.

28. Ozone UV spectroscopy. II. Absorption cross-sections and temperature dependence/ J. Malicet, D. Daumont, J. Charbonnier, C. Parisse, A. Chakir, J. Brion // Journal of Atmospheric Chemistry. - 1995. - V. 21. - Iss. 3. - P. 263-273.

29. The Huggins band of ozone: Assignment of hot bands / H. Zhu, Z.W. Qu, S.Y. Grebenshchikov, R. Schinke, J. Malicet, J. Brion, D. Daumont // Journal of Chemical Physics. - 2005. - V. 122. Iss. 2. - Article Number 024310.

30. Krueger A.J., Minzner R.A. Mid-latitude ozone model for the 1976 U.S. Standard Atmosphere // Journal of Geophysical Research. - 1976. - V. 81. - № D24. - P. 4477-4481.

Поступила 07.11.2018 2. 


\section{Информация об авторах}

Долгий С.И., кандидат физико-математических наук, старший научный сотрудник Центра лазерного зондирования атмосферы Института оптики атмосферы им. В.Е. Зуева Сибирского отделения Российской академии наук.

Невзоров $\boldsymbol{A}$.A., аспирант, младший научный сотрудник научно-образовательного центра Института оптики атмосферы им. В.Е. Зуева Сибирского отделения Российской академии наук.

Невзоров A.B., кандидат технических наук, старший научный сотрудник Центра лазерного зондирования атмосферы Института оптики атмосферы им. В.Е. Зуева Сибирского отделения Российской академии наук.

Романовский O.A., доктор физико-математических наук, заведующий научно-образовательным центром Института оптики атмосферы им. В.Е. Зуева Сибирского отделения Российской академии наук; профессор кафедры управления инновациями факультета инновационных технологий Национального исследовательского Томского государственного университета.

Харченко О.В., кандидат физико-математических наук, старший научный сотрудник центра лазерного зондирования атмосферы Института оптики атмосферы им. В.Е. Зуева Сибирского отделения Российской академии наук. 
UDC 551.510.532; 551.501.816

\title{
LIDAR FOR MEASUREMENT OF OZONE VERTICAL DISTRIBUTION IN THE ATMOSPHERE
}

\section{Sergey I. Dolgii',}

dolgii@iao.ru

\author{
Alexey A. Nevzorov', \\ naa@iao.ru
}

\author{
Alexey V. Nevzorov", \\ nevzorov@iao.ru
}

\section{Oleg A. Romanovskii', roa@iao.ru}

\author{
Olga V. Kharchenko', \\ olya@iao.ru \\ 1 V.E. Zuev Institute of Atmospheric Optics SB RAS, \\ 1, Academician Zuev square, Tomsk, 634021, Russia. \\ ${ }^{2}$ National Research Tomsk State University, \\ 36, Lenin Avenue, Tomsk, 634050, Russia.
}

\begin{abstract}
One of the main climate-forming factors from the point of view of the radiation balance of the atmosphere are greenhouse gases, primarily ozone and gas components of the ozone cycles. Currently, operational information on their vertical distribution in the atmosphere can be obtained only using remote sensing, including laser one (lidar), which determines the relevance and scientific significance of this scientific and technical problem.

The main aim of the study is to develop a lidar for studies of vertical distribution of ozone in the upper troposphere-stratosphere in the altitude range (5-45) $\mathrm{km}$ at 299/341 and 308/353 nm in comparison with satellite measurements.

Methods: differential absorption lidar (DIAL).

Results. The paper introduces the lidar for measuring ozone vertical distribution above the city of Tomsk $(56,5 \mathrm{~N}, 85,0$ E). Lidar sounding of ozone was carried out on pairs of wavelengths 299/341 and 308/353 nm. The wavelengths used allow monitoring ozone vertical stratification within the upper troposphere, the stratosphere. The results of ozone lidar sensing as of January 2018 are given in comparison with the data of the satellites IASI/MetOp and AURA, as well as with the mid-latitude model of Kruger. The difference between the lidar profiles of ozone and the model ones is natural for the dynamics of the ozone layer on different observation days. The large differences in ozone vertical distribution in the values of lidar and the MetOp satellite at the altitudes of the stratosphere are explained by the need to correct the a priori model in the algorithm for reconstructing satellite data.

Conclusion. The lidar was developed to measure ozone profiles in the upper troposphere-the stratosphere. It allows reconstructing the vertical distribution of ozone in the altitude range (5-45) km at wavelengths 299/341 and 308/353 $\mathrm{nm}$.
\end{abstract}

Key words:

Lidar, ozone, atmosphere, differential absorption, MetOp satellite, AURA satellite.

The research was partially financially supported by the RF presidential scholarship for supporting young scientists and postgraduates (SP-3926.2018.3).

\section{REFERENCES}

1. Burlakov V.D., Dolgii S.I., Nevzorov A.A., Nevzorov A.V. Romanovskii O.A., Kharchenko O.V. Lidar sensing of ozone in the upper troposphere - lower stratosphere: technique and result of measurements. Bulletin of the Tomsk Polytechnic University. Geo Assets Engineering, 2015, vol. 326, no. 9, pp. 124-132. In Rus.

2. Weitkamp C. Lidar: range resolved optical remote sensing of the atmosphere. New York, Springer-Verlag New York Publ., 2005. $456 \mathrm{p}$.

3. Bobrovnikov S.M., Matvienko G.G., Romanovskii 0.A., Serikov I.B., Sukhanov A.Y. Lidarny spectroscopicheskiy gazoanalis atmosfery [Lidar spectroscopic gas analysis of the atmosphere]. Tomsk, IAO S0 RAN Publ., 2014. 510 p.

4. Balin Yu.S., Borovoy A.G., Burlakov V.D., Dolgii S.I., Klemasheva M.G., Konoshonkin A.V., Kokhanenko G.P., Kustov N.V., Marichev V.N., Matvienko G.G., Nevzorov A.A., Nevzorov A.V., Penner I., Romanovskiy 0.A., Samokhvalov S.V., Sukha- nov A.Ya., Kharchenko O.V., Shishko V.A. Lidarny monitoring oblachnykh $i$ aerozolnykh poley, malykh gasovykh sostavlayshchikh meteoparametrov atmosfery [Lidar monitoring of cloudy and aerosol fields, small gas components and meteoparameters of the atmosphere]. Tomsk, IA0 SO RAN Publ., 2015. $450 \mathrm{p}$.

5. Matvienko G.G., Balin Yu.S., Bobrovnikov S.M., Romanovskiy 0.A., Kokhanenko G.P., Samoylova S.V., Penner I., Gorlov E.V., Zharkov V.I., Sadovnikov S.A., Kharchenko O.V., Yakovlev S.V., Bazhenov O.E., Burlakov V.D., Dolgii S.I., Makeev A.P., Nevzorov A.A., Nevzorov A.V. Sibirskay lidarnaya stansiya: apparatura $i$ resultaty [Siberian lidar station: equipment and results]. Tomsk, IA0 S0 RAN Publ., 2016. 440 p.

6. Sugita T., Akiyoshi H., Wolfram E., Salvador J., Ohyama H., Mizuno A. Comparison of ozone profiles from DIAL, MLS, and chemical transport model simulations over Río Gallegos, Argentina, during the spring Antarctic vortex breakup, 2009. Atmospheric Measurement Techniques, 2017, vol. 10, Iss. 12, pp. 4947-4964. 
7. Duflot V., Baray J.-L., Payen G., Marquestaut N., Posny Fr., Metzger J.-M., Langerock B., Vigouroux C., Hadji-Lazaro J., Portafaix Th., De Mazière M., Coheur P.-Fr., Clerbaux C., Cammas J.-P. Tropospheric ozone profiles by DIAL at Maïdo Observatory (Reunion Island): system description, instrumental performance and result comparison with ozone external data set. At mospheric Measurement Techniques, 2017, vol. 10, Iss. 9, pp. 3359-3373.

8. Aggarwal M., Whiteway J., Seabrook J., Gray L., Strawbridge K., Liu P., O'Brien J., Li Sh.-M., McLaren R. Airborne lidar measurements of aerosol and ozone above the Canadian oil sands region. Atmospheric Measurement Techniques, 2018, vol. 11, Iss. 6, pp. 3829-3849.

9. Wang L., Newchurch M.J., Alvarez II R.J., Berkoff T.A., Brown S., Carrion W., De Young R.J., Johnson B.J., Ganoe R., Gronoff G., Kirgis G., Kuang Sh., Langford A.0., Leblanc T., McDuffie E.E., McGee T.J., Pliutau D., Senff C.J., Sullivan J.T., Sumnicht G., Twigg L.W., Weinheimer A.J. Quantifying TOLNet ozone lidar accuracy during the 2014 DISCOVER-AQ and FRAPPÉ campaigns. Atmospheric Measurement Techniques, 2017, vol. 10, Iss. 10, pp. 3865-3876.

10. Park Ch.B., Nakane H., Sugimoto N., Matsui Ich., Sasano Y., Fujinuma Y., Ikeuchi Iz., Kurokawa J.-Ich., Furuhashi N. Algorithm improvement and validation of National Institute for Environmental Studies ozone differential absorption lidar at the Tsukuba Network for Detection of Stratospheric Change complementary station. Applied Optics, 2006, vol. 45, no. 15, pp. 3561-3576.

11. Nakazato M., Nagai T., Sakai T., Hirose Y. Tropospheric ozone differential-absorption lidar using stimulated Raman scattering in carbon dioxide. Applied Optics, 2007, vol. 46, no. 12, pp. 2269-2279.

12. Godin-Beekmanna S., Songa T., Heeseb B. Long-term DIAL monitoring of the stratosphericozone vertical distribution. Proceedings of SPIE, 2003, vol. 4893, pp. 251-263.

13. Gaudel A., Ancellet G., Godin-Beekmann S. Analysis of 20 years of tropospheric ozone vertical profiles by lidar and ECC at Observatoire de Haute Provence (OHP) at 44N, 6.7E. Atmospheric Environment, 2015, vol. 113, pp. 78-89.

14. McDermid I.S., Godin S.M., Lindquist L.0. Ground-based laser DIAL system for long-term measurements of stratospheric ozone. Applied Optics, 1990, vol. 29, no. 25, pp. 3603-3612.

15. McDermid I.S., Beyerle G., Haner D.A., Leblanc T. Redesign and improved performance of the tropospheric ozone lidar at the Jet Propulsion Laboratory Table Mountain Facility. Applied Optics, 2002, vol. 41, no. 36, pp. 7550-7555.

16. Steinbrecht W., McGee T.J., Twigg L.W., Claude H., Sch“”onenborn F., Sumnicht G.K., Silbert D. Intercomparison of stratospheric ozone and temperature profiles during the October 2005 Hohenpeißenberg Ozone Profiling Experiment (HOPE). Atmospheric Measurement Techniques, 2009, no. 2, Iss. 1, pp. $125-145$.

17. Sullivan J.T., McGee T.J., Sumnicht G.K., Twigg L.W., Hoff R.M. A mobile differential absorption lidar to measure subhourly fluctuation of tropospheric ozone profiles in the Baltimo-
re-Washington, D.C. region. Atmospheric Measurement Techniques, 2014, vol. 7, Iss. 10, pp. 3529-3548.

18. Pavlov A.N., Stolyarchuk S.Yu., Shmirko K.A., Bukin O.A. Lidar Measurements of Variability of the Vertical Ozone Distribution Caused by the Stratosphere-Troposphere Exchange in the Far East Region. Atmospheric and Oceanic Optics, 2013, vol. 26, no. 2, pp. 126-134.

19. Burlakov V.D., Dolgii S.I., Nevzorov A.V. Modernizatsiay izmeritelnogo kompleksa Sibirskoy lidarnoy stansii [Modification of the measuring complex at the Siberian Lidar Station]. Atmospheric and oceanic optics, 2004, vol. 5, no. 10, pp. 1022-1027.

20. Dolgii S.I., Nevzorov A.A., Nevzorov A.V., Romanovskii O.A., Kharchenko 0.V. Intercomparison of ozone vertical profile measurements by differential absorption lidar and IASI/Met0p satellite in the upper troposphere-lower stratosphere. Remote Sensing, 2017, vol. 9, no. 5, pp.447-462.

21. MLS data documentation. Available at: https://mls.jpl.nasa.gov/data/datadocs.php (accessed 30 July 18).

22. Goddard Space Flight Center. Dannie sputnika AURA (MLS) [AURA (MLS) data]. Available at: https://avdc.gsfc.nasa.gov/pub/data/satellite/Aura/MLS/V04/L2GPOVP/03/ (accessed 30 July 18).

23. August T., Klaes D., Schlüssel P., Hultberg T., Crapeau M., Arriaga A., O'Carroll A., Coppens D., Munro R., Calbet X. IASI on Metop-A: Operational Level 2 retrievals after five years in orbit. Journal of Quantitative Spectroscopy and Radiative Transfer, 2012, vol. 113, no. 11, pp. 1340-1371.

24. Mezheris R. Laser remote sensing. Moscow, World Publ., 1987. $552 \mathrm{p}$.

25. Gorshelev V., Serdyuchenko A., Weber M., Chehade W., Burrows J.P. High spectral resolution ozone absorption cross-sections - Part 1: Measurements, data analysis and comparison with previous measurements around $293 \mathrm{~K}$. Atmospheric Measurement Techniques, 2014, vol. 7, Iss. 2, pp. 609-624.

26. Serdyuchenko A., Gorshelev V., Weber M., Chehade W., Burrows J.P. High spectral resolution ozone absorption cross-sections - Part 2: Temperature dependence. Atmospheric Measurement Techniques, 2014, vol. 7, Iss. 2, pp. 625-636.

27. El'nikov A.V., Zuev V.V. Bifrequency laser sounding of stratospheric ozone under conditions of high degree of aerosol loading. Atmospheric and oceanic optics, 1992, vol. 5, no. 10, pp. 681-683.

28. Malicet J., Daumont D., Charbonnier J., Parisse C., Chakir A., Brion J. Ozone UV spectroscopy. II. Absorption cross-sections and temperature dependence. Journal of Atmospheric Chemistry, 1995, vol. 21, Iss. 3, pp. 263-273.

29. Zhu H., Qu Z.W., Grebenshchikov S.Y., Schinke R., Malicet J., Brion J., Daumont D. The Huggins band of ozone: Assignment of hot bands. Journal of Chemical Physics, 2005, vol. 122, Iss. 2, article number 024310 .

30. Krueger A.J., Minzner R.A. Mid-latitude ozone model for the 1976 U.S. Standard Atmosphere. Journal of Geophysical Research, 1976, vol. 81, no. D24, pp. 4477-4481

Received: 7 November 2018.

\section{Information about the authors}

Sergey I. Dolgii, Cand. Sc., senior researcher, V.E. Zuev Institute of Atmospheric Optics SB RAS.

Alexey A. Nevzorov, junior researcher, V.E. Zuev Institute of Atmospheric Optics SB RAS; postgraduate, National Research Tomsk State University.

Alexey V. Nevzorov, Cand. Sc., senior researcher, V.E. Zuev Institute of Atmospheric Optics SB RAS.

Oleg A. Romanovskii, Dr. Sc., head of the Research and Educational Centre, V.E. Zuev Institute of Atmospheric Optics SB RAS; National Research Tomsk State University.

Olga V. Kharchenko, Cand. Sc., senior researcher, V.E. Zuev Institute of Atmospheric Optics SB RAS. 